\title{
Kritisch-reflexive Urteilskompetenz in der Bildungsarbeit
}

\author{
Konfliktsensible und rassismuskritische Perspektiven
}

Bildungsprozesse im Kontext der Sozialen Arbeit sind niemals neutral. Gerade in konfliktiven und diversen Gesellschaften kann non-formale Bildung vielfältige negative Nebenwirkungen mit sich bringen. Wie kann es daher gelingen, eine diversitäts- und konfliktsensible kritisch-reflexive Urteilskompetenz von Bildungsverantwortlichen zu erhöhen, um diese negative Nebenwirkungen zu minimieren?

$\mathrm{D}$ er vorliegende Beitrag basiert auf zwei Grundannahmen: Bildungsprozesse sind niemals neutral und in der hochkomplexen diversifizierten Weltgesellschaft extrem konfliktanfällig und spannungsreich. Damit ist gemeint, dass durch Bildungsangebote, -strukturen, -formate und -materialien oftmals ungewollt vorhandene Konfliktpotentiale verstärkt, Diskriminierungs-

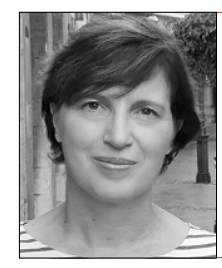

\section{Susanne Bücken}

Katholische Hochschule Nordrhein-Westfalen, Aachen, Deutschland

Sozialpädagogin, Lehrdozentin und Bildungswissenschaftlerin.

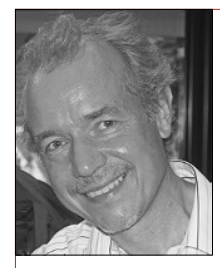

Norbert Frieters-Reermann

Katholische Hochschule Nordrhein-Westfalen Katholische Fachhochschule gGmbH,

Aachen, Deutschland

*1970, Dr., Professor für Theorien und Konzepte Sozialer Arbeit an der Katholischen Hochschule NRW.

n.frieters-reermann@katho-nrw.de

Zusammenfassung Bildungsprozesse sind niemals neutral und in der hochkomplexen diversifizierten Weltgesellschaft extrem konfliktanfällig und spannungsreich. Damit wird angesprochen, dass durch Bildungsangebote, -strukturen, -formate und -materialien oftmals ungewollt vorhandene Konfliktpotentiale verstärkt, Diskriminierungstendenzen verschärft und Exklusionsdynamiken erhöht werden. Von daher ist eine umfassende kritisch-reflexive Urteilskompetenz aller verantwortlichen Akteur"innen von zentraler Bedeutung. Diese wird im vorliegenden Beitrag aus der Perspektive einer konfliktsensiblen und rassismuskritischen Bildung hergeleitet.

Schlüsselwörter Rassismuskritik, Diversität, Konfliktsensibilität, Kritisch-reflexive Urteilskompetenz tendenzen verschärft und Exklusionsdynamiken erhöht werden. Von solchen nicht intendierten negativen Wirkungen sind ebenso extracurriculare, außerschulische und non-formale Bildungsbereiche betroffen und damit potenziell auch alle Bildungsprozesse im Kontext der Sozialen Arbeit. Diese sich zunehmend durchsetzende Erkenntnis erfordert es, kontinuierlich die scheinbar per se inklusive, friedensstiftende und gewaltfreie Kraft von Bildung zu entmystifizieren und zu dekonstruieren. Dass Bildung nämlich „grundsätzlich zur Förderung der zwischenmenschlichen Kooperation und Verständigung, zur Stärkung des sozialen Zusammenhaltes, zum Abbau sozialer Ungleichheiten und zur moralischen Verbesserung des Menschen beiträgt, zählt zu den einflussreichen Trugbildern und Selbsttäuschungen der Pädagogik. " (Seitz 2004, S. 49). Von daher rücken seit einigen Jahren auch zunehmend die negativen, gewaltfördernden, diskriminierenden und ambivalenten Aspekte von Bildung und Erziehung in den Fokus der Wissenschaft (Seitz 2004; INEE 2010; Frieters-Reermann 2015; Castro Varela 2015).

Uns geht es also darum, Annahmen von Bildung als Kritik mit einer Kritik der Bildung zusammenzudenken (vgl. Hartmann 2013, S. 254) und Anregungen für die Entwicklung einer kritisch-reflexiven Urteilskompetenz zu entwerfen. Gerade vor dem Hintergrund von migrationsgesellschaftlichen Transformationsprozessen und damit verbundener „Rekonstruktion und Neubewertung von Fragen nach politischer und sozialer Gerechtigkeit“ (vgl. Lange et al. 2018, S. 1) wird ersichtlich, dass Bildungsprozesse in Spannungsverhältnisse verortet sind und dass sich die hieraus ergebenden Herausforderungen "gegenseitig bedingen und eng miteinander verzahnt sind." (ebd.)

Aus diesem Blickwinkel verstehen wir unter professioneller kritisch-reflexiver Urteilskompetenz eine politisch- 


\section{Extrablick: Urteilsbildung in der Sozialen Arbeit}

ethische Urteilsbildung, wie sie auch als Bildungsziel in der politischen Bildung thematisiert wird und welche eine Reflexion auf die eigenen Urteilsmaßstäbe (vgl. May 2017, S. 15) notwendigerweise immer mit einbezieht und voraussetzt. Hiermit fokussieren wir eine kritisch-reflexive Professionalität, die über ein diagnostisches Fallverstehen und über die Reflexion von Machtkonstellationen innerhalb der analytisch-diagnostischen Prozesse hinausgeht und die nicht allein soziale Verhältnisse in den Fokus nimmt, die gewaltbesetzte Problemlagen reproduzieren (vgl. Otto 1973, S. 256). Wir interessieren uns für bildungstheoretische und -praktische Ansätze zur kritisch-reflexiven Urteilsbildung, die immer auch die eigenen Gewaltpotentiale und Machtverhältnisse reflektieren. Mit diesem Anliegen werden wir im Folgenden die Bedeutung einer kritisch-reflexiven Urteilskompetenz für die Bildungskontexte und die Soziale Arbeit hervorheben und konkrete Handlungsoptionen skizzieren. Dabei interessieren uns Urteilsmaßstäbe einer professionellen kritisch-reflexiven Urteilskompetenz, die wir anhand von zwei theoretisch unterschiedlich gefassten Zugängen,

1. dem Do not Harm-Ansatz und

2. dem rassismuskritischen Ansatz,

\section{diskutieren.}

\section{Anregungen aus der internationalen Entwicklungszusammenarbeit ${ }^{1}$}

Ein zentraler Ausgangs- und Bezugspunkt für die Entwicklung konfliktsensibler Bildungsansätze sind die Erfahrungen der Entwicklungszusammenarbeit (und der humanitären Hilfe) im Kontext von massiven innerstaatlichen Gewalteskalationen in den 1990er-Jahren u. a. in Somalia, in Rwanda und im ehemaligen Jugoslawien. In diesen Kontexten wurden zum ersten Mal systematisch die Verstrickungen und Wechselwirkungen zwischen internationalen (Hilfs)Organisationen einerseits und den nationalen und innergesellschaftlichen Gewalt- und Konfliktdynamiken andererseits analysiert. Dadurch wurde das Selbstbild der internationalen Entwicklungszusammenarbeit per se friedensfördernde, stabilisierende und positive Wirkungen zu entfalten, tief erschüttert. Die damit verbundene Kränkung führte u. a. zu der Forderung nach Do No Harm („Richte keinen Schaden an“), die durch Anderson (1999) in den internationalen Diskurs eingeführt wurde und mittlerweile zu einem international anerkannten Qualitätsstandard konfliktsensibler Entwicklungszusammenarbeit geworden ist (CDA 2004; OECD 2010; Goddard und Annaraj 2017).

Gemeint ist, dass die Organisationen der Entwicklungszusammenarbeit sich kontinuierlich kritisch-re- flexiv mit der eigenen Rolle in akuten Gewalt- und Konfliktdynamiken auseinandersetzen und mögliche konfliktverschärfende Tendenzen frühzeitig erkennen und entsprechend versuchen, ihre Aktions-, Kommunikations- und Kooperationsweisen anzupassen. Auf der Basis der Pionierarbeiten von Anderson und dem internationalen Do-no-harm-Netzwerk (CDA) hat sich der Diskurs um konfliktsensible Planungs- und Steuerungsinstrumente in der Entwicklungszusammenarbeit rasant entwickelt und wurde explizit für Bildungsprozesse und -systeme in Gewalt- und Konfliktkontexten ausgeweitet (vgl. INEE 2010). Dabei geht es bei allen Konzepten und Ansätzen im Kern darum, dass die professionellen Fachkräfte kritisch-reflexiv die intendierten und nicht intendierten, die direkten und indirekten sowie die negativen und positiven Wirkungen entwicklungspolitischer und bildungsbezogener Maßnahmen auf den Konfliktverlauf erfassen, bewerten und entsprechend handeln. Um umgekehrt gilt es, die Auswirkungen des Konfliktes auf die Interventionen der Entwicklungszusammenarbeit und auf Bildungsprozessen in den Blick zu nehmen. Für die Befähigung von Fachkräften zur kritisch-reflexiven Urteilskompetenz in Bildungskontexten lohnt es sich, auf die Pionierarbeiten von Anderson und den damit verbundenen Do-no-harm-Framework zurückzugreifen. Denn auch wenn dieser vor dem Hintergrund akuter und eskalierenden Gewaltdynamiken entwickelt wurde, eröffnet er ein hohes Anregungspotential für alle Bildungsprozesse in einer konfliktanfälligen Weltgesellschaft.

Anderson hat auf der Basis ihrer Forschungen sieben Lektionen (Lessons Learned) abgeleitet, durch die eine konfliktsensible und selbstreflexive Haltung und Praxis von Fachkräften und Institutionen der Entwicklungszusammenarbeit gestärkt werden könnte (Anderson 1999; CDA 2004). Diese werden nachfolgend aufgegriffen und für die kritisch-reflexive Urteilskompetenz in Bildungskontexten weitergedacht.

- Lektion 1: Bildungsprozesse sind niemals neutral, sondern stehen immer in Wechselwirkung zu den gesellschaftlichen Konfliktdynamiken; von daher hat jede Bildungspraxis Auswirkungen auf den Konflikt.

- Lektion 2: Jeder Konfliktkontext wird durch zwei Arten von Faktoren charakterisiert: Dividers (Trennende Faktoren mit Eskalations- und Spaltungspotentialen) und Connectors (Verbindende Faktoren mit Deeskalations- und Kooperationspotentialen).

- Lektion 3: Jeder Bildungsprozess hat direkte oder indirekte Auswirkungen auf die beide Faktorengruppen (Dividers und Connectors) sowohl in positiver konfliktentschärfender als auch in negativer konfliktverschärfender Weise. 
- Lektion 4: Alle expliziten mit dem Bildungsprozesse einhergehenden oder auf diesen basierenden Dynamiken, z. B. strukturelle Rahmenbedingungen, institutionelle Zugangsmöglichkeiten, administrative Regelungen und organisationale Abläufe wirken auf den Konfliktkontext ein.

- Lektion 5: Alle impliziten mit dem Bildungsprozesse einhergehenden oder auf diesen basierenden Dynamiken z. B. ethische Werthaltungen, verdeckte Botschaften und bewusste oder unbewusste normative Sichtweisen wirken auf eine andere Art auf den Konfliktkontext ein.

- Lektion 6: Jedes Detail im Kontext von Bildungsprozessen (in Bezug auf die expliziten und impliziten Dynamiken) ist für eine konfliktsensible Ausrichtung von Bedeutung.

- Lektion 7: Für jeden Bildungsprozess gibt es Optionen für alternative Vorgehensweisen, um negative konfliktverschärfende Auswirkungen zu reduzieren und positive konfliktentschärfende zu erhöhen.

Diese sieben für den Bildungskontext abgewandelten Do-no-harm-Lektionen sind aus unserer Sicht für die Stärkung einer kritisch-reflexiven Urteilskompetenz von Fachkräften der Sozialen Arbeit, die in Bildungskontexten arbeiten in hohem Maße anschlussfähig. Im Kern geht es darum, eine professionelle Haltung zu entwickeln, die sich die eigenen Verstrickungen mit den Konfliktdynamiken und den Dividers und Connectors immer wieder bewusstmacht. Auf dieser Basis sollten Fachkräfte sich fortlaufend fragen, von welchen möglicherweise exkludierenden, diskriminierenden und potenziell konfliktverschärfenden strukturellen Rahmenbedingungen und ethischen Werthaltungen und Botschaften ihr Handeln und ihre Einrichtung geprägt sind. Auf der Basis dieser kritischen Überprüfung der eigenen Bildungsstrukturen, -praktiken und -inhalte können entsprechende Umorientierungen erfolgen, um Bildungsprozesse möglichst konfliktsensibel auszurichten und die negativen und destruktiven Wirkungen weitreichend und weitestgehend zu reduzieren.

\section{Machtkritik und Ermutigung}

Worüber sprechen wir, wenn wir eine kritisch-reflexive Urteilskompetenz mit einer rassismuskritischen Perspektive für die Bildungsarbeit und Soziale Arbeit verbinden? Mit den folgenden Überlegungen zu einer rassismuskritischen Perspektive wird über eine kritisch-reflexive Urteilskompetenz nachgedacht, die eine politisch-ethische Positionierung einbezieht und die jedes national gedachte Paradigma einer Reflexion auf Bildungsprozesse in Frage stellt. Mit diesem Bildungsanliegen sprechen wir konkret eine rassismuskritisch-reflexive Urteilkompetenz an, die wir allerdings nicht als eine, Sonderkompetenz' betrachten, sondern als Bestandteil jeder kritisch-reflexiven Professionalisierung für die Bildungsarbeit und Soziale Arbeit. Hierzu greifen wir für die folgenden Überlegungen Kriterien für die politische Bildung auf, die in der „Frankfurter Erklärung für eine kritisch-emanzipatorische Politische Bildung “ (2015) vorgestellt werden. In dieser werden ausgehend von gesellschaftlichen Umbrüchen und Krisen, Kontroversität, Machtkritik, Reflexivität und Ermutigung für die politische Bildungsarbeit eingefordert. Es wird deutlich gemacht, dass gesellschaftliche Konflikte, die von Interessensgegensätzen und Macht- und Herrschaftsverhältnissen durchzogen sind, die Wahrnehmung und Analyse von intersektional verwobenen sozialen Ungleichheiten, Machtasymmetrien und Abhängigkeiten erforderlich machen und diese Thematisierungen und Analysen bedeutungsvolle Voraussetzung dafür sind, um sich ernstgemeint dem „Ansinnen der Demokratisierung gesellschaftlicher Verhältnisse" (vgl. ebd.) anzunähern.

Eines dieser gesellschaftlichen Macht- und Herrschaftsverhältnisse ist Rassismus, der mit biologistischen, kulturalistischen und geopolitischen Bezugnahmen an Rassekonstruktionen anknüpft und soziale Ausgrenzung normalisiert (vgl. Terkessides 2004, S. 98ff.). „Rassistische und an Rassismus anschließende Unterscheidungen zwischen dem natio-ethno-kulturell kodierten Wir und Nicht-Wir, die beispielsweise über mediale Berichterstattung, Handlungsroutinen oder rechtliche Praktiken aufgerufen oder angeeignet werden, stehen auf allen gesellschaftlichen Ebenen (individuell, strukturell, institutionell usw.) als potenzielle Deutungs- und Handlungsressourcen zur Verfügung. “ (Kooroshy et al. 2018, S. 22) Für einen macht- und rassismuskritischen Erkenntnis-, Verstehens und Urteilsprozess in der Bildungsarbeit und der Sozialen Arbeit ist demnach eine „radikale Reflexivität“ (Kessl/Maurer 2012, S. 43f.) gefragt, die an der individuellen, institutionellen und strukturellen Involviertheit in Macht- und Herrschaftsverhältnissen ansetzt und thematisiert, dass Bildungsprozesse und Lernverhältnisse in rassistische und rassifizierte Verhältnisse eingewoben sind. In Anlehnung an Überlegungen zur politischen Urteilsbildung deuten wir die Entwicklung einer rassismuskritisch-reflexiven Urteilskompetenz in der Bildungsarbeit und Sozialen Arbeit als einen subjektorientierten Bildungsprozess, für den es von Relevanz ist, die eigenen migrationsgesellschaftlichen Maßstäbe der Urteilsbildung kontinuierlich einer kritischen Reflexion zu unterziehen (vgl. May 2017, S. 15), da migrationsgesellschaftliche Maßstäbe des Urteilens natio-ethno-kulturelle kodierte Zugehörigkeitsordnungen bezugnehmend auf Konzepte von Nation, Ethnie/Ethnizität, Kultur und Religion vermitteln (vgl. Mecheril et al. 2020, S. 7). 


\section{Extrablick: Urteilsbildung in der Sozialen Arbeit}

Soziale Arbeit und Bildungsprozesse sind in migrationspolitische und -gesellschaftliche Diskurse eingebunden, die rassistisch strukturiert sind. Es ist demnach auch für die Professionellen, wie Bildungsarbeiter_innen, Sozialarbeiter_innen, Lehrenden und Wissenschaftler_innen nicht möglich, sich außerhalb von rassistischen Strukturen und Diskursen zu verhalten. Für die (Bildungs-) Praxis der Sozialen Arbeit ist eine rassismuskritisch-reflexive Urteilskompetenz bislang allerdings wenig ausgeprägt. Denn auch, wenn in Folge der mörderischen Attentate in Halle und Hanau sowie der globalen Proteste der BlackLives-Matter-Bewegung die strukturelle Dimension von Rassismus gegenwärtig stärker in den Fokus rückt, wird Rassismus in seinen alltäglichen und institutionellen Ausprägungen nicht oder wenig wahrgenommen und weiterhin zumeist individualisiert, bagatellisiert, naturalisiert und pathologisiert (vgl. Melter 2015, S. 16).

Das Ausblenden der Involviertheit in die Normalität von Rassismus betrifft gerade auch die Soziale Arbeit, die mit ihrer grundlegenden Logik sozialdiagnostischer Praxis, „Unterscheiden - Identifizieren - Behandeln“ (Mecheril und Melter 2010, S. 124), immer auch daran beteiligt, gesellschaftliche Ordnungen der Zugehörigkeit zu entwerfen, zu produzieren und zu bestätigen, wie im Sprechen zum ,Migrationshintergrund', zum ,Islam‘, zum ,Deutschsein', zum ,Nicht-Deutschsein'.

Aus einer rassismuskritischen Perspektive verweisen diese Konstruktionen ,der Anderen', in Verschränkung mit dominanzkulturellen Vorstellungen von Anpassung und Integration, auf ein Desiderat einer rassismus- und machkritischen Wissensbildung in der Sozialen Arbeit. Solche Leerstellen formen eine marginal ausgebildete rassismuskritisch-reflexive Urteilskompetenz und erzeugen Denk- und Handlungsweisen, in denen „Soziale Arbeit als Komplizin eines regulierenden Staates“ auftritt, „der seit seiner Entstehung bis in die Gegenwart mit race verwoben ist“ (Kooroshy et al. 2018, S. 22). Insbesondere wird die Abwesenheit einer rassismuskritischreflexiven Professionalität in Gestalt eines sekundären Rassismus ersichtlich, den Melter auf individueller Ebene als eine ausgeprägte Abwehrhaltung von Professionellen in der Kinder- und Jugendhilfe analysiert, sich mit dem Thema und der alltäglichen Realität von Rassismus auseinander zu setzen (vgl. Melter 2007, S. 119). Dementgegen werden Rassismuserfahrungen viel eher bagatellisiert und Infrage gestellt, das eigene emotionale Erleben der von Rassismus betroffenen Kindern und Jugendlichen häufig delegitimiert sowie als unangemessener Rassismusvorwurf entwertet (vgl. ebd.).

Für die Entwicklung einer rassismuskritisch-reflexiven Urteilkompetenz bedarf es also der Analyse und Kritik der eigenen Wissensvermittlung und Wissensprodukti- on und des Versuchs, „Lernen in Zusammenhang mit Begehren, Macht und Herrschaft zu denken" (Castro Varela 2015, S. 347). Mit dieser Perspektive interessieren Fragen dazu, „Was wird von wem vermittelt? Wie werden die Zielgruppen imaginiert und repräsentiert? “ und warum wird „die affektive und auch die körperlichmaterielle Seite von Bildung vernachlässigt.“ (ebd.) Es bedarf einer kritischen Perspektive auf die für ,wahr" befundenen Wissensbestände einer weißen und eurozentrischen Wissensproduktion, die mit ,prominenten Referenzfiguren der Aufklärung “ (Kooroshy et al. 2018, S. 23), wie ,Emanzipation', ,Autonomie“ ,Menschenrechte' für die Soziale Arbeit konstitutiv ist (vgl. ebd.). Die Entwicklung einer (rassismus)kritischen Reflexivität verstehen wir ausdrücklich auch als eine Ermutigung für Bildungsarbeiter*innen und Sozialarbeiter*innen, „Macht- und Ohnmachtserfahrungen zu thematisieren und zu hinterfragen“ (Frankfurter Erklärung 2025), diskriminierende Repräsentationsverhältnisse offen zu legen und „solidarischen Beistand“ (Stojanov 2014, S. 108) zu leisten: „Gerade weil Soziale Arbeit herrschende Verhältnisse und dominante Diskurs festigen kann, ist sie auch in der Lage, diese zu irritieren." (Attia 2013, S. 335).

Eingegangen. 7. April 2021

Angenommen. 11. Mai 2021

Funding. Open Access funding enabled and organized by Projekt DEAL.

Open Access. Dieser Artikel wird unter der Creative Commons Namensnennung 4.0 International Lizenz veröffentlicht, welche die Nutzung, Vervielfältigung, Bearbeitung, Verbreitung und Wiedergabe in jeglichem Medium und Format erlaubt, sofern Sie den/die ursprünglichen Autor(en) und die Quelle ordnungsgemäß nennen, einen Link zur Creative Commons Lizenz beifügen und angeben, ob Änderungen vorgenommen wurden.

Die in diesem Artikel enthaltenen Bilder und sonstiges Drittmaterial unterliegen ebenfalls der genannten Creative Commons Lizenz, sofern sich aus der Abbildungslegende nichts anderes ergibt. Sofern das betreffende Material nicht unter der genannten Creative Commons Lizenz steht und die betreffende Handlung nicht nach gesetzlichen Vorschriften erlaubt ist, ist für die oben aufgeführten Weiterverwendungen des Materials die Einwilligung des jeweiligen Rechteinhabers einzuholen.

Weitere Details zur Lizenz entnehmen Sie bitte der Lizenzinformation auf http://creativecommons.org/licenses/by/4.0/deed.de.

1. Den Autor*innen ist bewusst, dass der Begriff „Entwicklungszusammenarbeit" eurozentrisch-westlich konnotiert ist und somit stigmatisierende Tendenzen enthält. Mit dieser (selbst)kritischen Reflexion nutzen wir ihn als Terminus technicus, da er den Kontext, in dem konfliktsensible Ansätze systematisch erarbeitet und weiterentwickelt wurden, beschreibt. 


\section{Literatur}

(2015). Frankfurter Erklärung für eine kritische-emanzipatorische Politische Bildung. https://sozarb.h-da.de/fileadmin/documents/Fachbereiche/Soziale_Arbeit/Politische_Jugendbildung/Dokumente/Frankfurter_Erklaerung. pdf. Zugegriffen: 15. März 2021.

Anderson, M. B. (1999). Do no harm: how aid support peace-or war. Boulder: Lynne Rienner Publishers.

Attia, I. (2013). Perspektivwechsel durch Dekonstruktion. Islamdiskurs und rassismuskritische Soziale Arbeit. In B. Hünersdorf \& J. Hartmann (Hrsg.), Was ist und wozu betreiben wir Kritik in der Sozialen Arbeit? Disziplinäre und interdisziplinäre Diskurse (S. 333-350). Wiesbaden: VSSpringer.

Castro Varela, M. M. (2015). Überdeterminiert und reichlich komplex. Überlegungen zu Politischer Bildung im Kontext von Postkolonialismus und Postnazismus. In A. Hechler \& O. Stuve (Hrsg.), Geschlechterreflektierte Pädagogik gegen Rechts (S. 343-364). Opladen: In.

CDA Collaborative Learning Projects (2004). The do no harm framework for analyzing the impact of assistance on conflict: a handbook. Cambridge: CDA Collaborative Learning Projects.

Frieters-Reermann, N. (2015). Friedenspädagogik als Teil gewaltsensibler Bildung - oder umgekehrt? Denkanstöße aus der konfliktsensiblen Entwicklungszusammenarbeit. In N. Frieters-Reermann \& G. Lang-Wojtasik (Hrsg.), Friedenspädagogik und Gewaltfreiheit. Denkanstöße für eine differenzsensible Kommunikations- und Konfliktkultur. Leverkusen: Verlag Barbara Budrich.

Goddard, N., \& Annaraj, D. (2017). Conflict sensitivity meta-trends analysis. https://www.cdacollaborative.org/publication/getting-do-no-harm-tostick-successes-failures-and-varied-approaches. Zugegriffen: 10. Febr. 2021.

Hartmann, J. (2013). Bildung als kritisch-dekonstruktives Projekt - pädagogische Ansprüche und queere Einsprüche. In B. Hünersdorf \& J. Hartmann (Hrsg.), Was ist und wozu betreiben wir Kritik in der Sozialen Arbeit? Disziplinäre und interdisziplinäre Diskurse (S. 149-164). Wiesbaden: VS-Springer.

INEE (Inter-Agency Network for Education in Emergencies) (2010). INEE minimum standards for education: preparedness, response. https:// inee.org/resources/inee-minimum-standards. Zugegriffen: 10. Febr. 2021

Kessl, F., \& Maurer, S. (2012). Radikale Reflexivität als zentrale Dimension eines kritischen Wissenschaftsverständnisses Sozialer Arbeit. In E. Schimpf \& J. Stehr (Hrsg.), Kritisches Forschen in der Sozialen Arbeit. Gegenstandsbereiche - Kontextbedingungen - Positionierungen - Perspektiven (S. 43-56). Wiesbaden: VS-Springer.

Kooroshy, S., Mecheril, P., \& Shure, S. (2018). Rassismus in der Migrationsgesellschaft. In J. Wetzel \& C. Hunner-Kreisel (Hrsg.), Rassismuskritik in der Sozialen Arbeit und Rassismuskritik als Querschnittanfgabe (S. 1526). Lahnstein: Verlag neue praxis.

Lange, D., Martínez Calero, M., \& Rohloff, S. A. (2018). Einleitung. In S. A. Rohloff, M. Martínez Calero \& D. Lange (Hrsg.), Soziale Arbeit und Politische Bildung in der Migrationsgesellschaft (S. 1-4). Wiesbaden: VSSpringer.

May, M. (2017). Politische Urteilsbildung unter Druck. Polis, 3/2017, 13-15

Mecheril, P., \& Melter, C. (2010). Differenz und Soziale Arbeit. In F. Kessl \& M. Plößer (Hrsg.), Differenzierung, Normalisierung, Andersheit. Soziale Arbeit als Arbeit mit dem Anderen (S. 117-131). Wiesbaden: VS-Springer.

Mecheril, P., Bücken, S., Streicher, N., \& Velho, A. (2020). Einleitung. In S. Bücken, N. Streicher, A. Velho \& P. Mecheril (Hrsg.), Migrationsgesellschaftliche Diskriminierungsverhältnisse in Bildungssettings. Analysen, Re flexionen, Kritik (S. 1-17). Wiesbaden: VS-Springer.

Melter, C. (2007). Sekundärer Rassismus in der Sozialen Arbeit. In T. Geisen \& C. Riegel (Hrsg.), Jugend, Partizipation und Migration. Orientierungen im Kontext von Integration und Ausgrenzung (S. 107-128). Wiesbaden: VS-Springer.

Melter, C. (2015). Diskriminierungs- und rassismuskritische Soziale Arbeit im postkolonialen und postnationalistischen Deutschland?! Einleitende Überlegungen. In C. Melter (Hrsg.), Diskriminierungs- und rassismuskritische Soziale Arbeit und Bildung, Praktische Herausforderungen, Rabmungen und Reflexionen (S. 7-19). Weinheim, Basel: Beltz.

OECD (2010). Do no harm. International Support für Statebuilding. Paris: OECD.
Otto, H.-U. (1973). Professionalisierung und gesellschaftliche Neuorientierung - Zur Transformation beruflichen Handelns in der Sozialarbeit. In H.-U. Otto \& S. Schneider (Hrsg.), Gesellschaftliche Perspektiven der Sozialarbeit (S. 247-263). Neuwied: Luchterhand.

Seitz, K. (2004). Bildung und Konflikt. (Studie für die GTZ). Eschborn: Deutsche Gesellschaft für Technische Zusammenarbeit (GTZ) GmbH.

Stojanov, K. (2014). Solidarität und Bildung in der Migrationsgesellschaft. In A. Broden \& P. Mecheril (Hrsg.), Solidarität in der Migrationsgesellschaft. Befragung einer normativen Grundlage (S. 95-109). Bielefeld: transcript.

Terkessides, M. (2004). Die Banalität des Rassismus. Migranten zweiter Generation entwickeln eine neue Perspektive. Bielefeld: transcript. 